Journal of Engineering and Applied Sciences 14 (14): 4795-4803, 2019

ISSN: 1816-949X

(C) Medwell Journals, 2019

\title{
Comparison Between Bat Algorithm and Harmony Algorithm using Retina Authentication
}

\author{
Iraq Ali Hussein and Ziyad Tariq Mustafa Al-Ta'i \\ Department of Computer Science, Collage of Science, University of Diyala, Diyala, Iraq
}

\begin{abstract}
Retina authentication technology's captures and analyzes them patterns off vascular on them thins nerve on the backs of them eyeball that processes lights entering through the pupil. Retinal patterns are highly distinctive traits; It cannot begotten or stolen. No, two people have same retinal pattern and the features extracted from retinal image are the most stable and reliable than other biometrics features. Due to these reasons the retina is chosen as robust source in the proposed authentication systems. In this thesis, a system for recognition based on vascular pattern of human retina is introduced. This system contains three stages (preprocessing, features extracting and matching). Preprocessing stage is used in order to make retina image more clear by using Gaussian and Canny filters and Haar wavelet transformer. Features extracting phase is done using two types of inspired algorithms (Bat and Harmony). Each extracted feature list is assembled as a feature vector which is used in order to distinguish between different retinal images. Finally, the discrimination analysis of features is evaluated in matching phase by using Euclidean distance measure. The results from this research declared that the best inspired algorithms for the proposed retinal authentication system are harmony algorithm in which the obtained accuracy is $(100 \%)$ while the accuracy of bat algorithm $(98.65 \%)$. However, harmony algorithm is the best for the proposed authentication system in time consuming which is $(3.0 \mathrm{sec})$ and bat consuming time is $(7.3 \mathrm{sec})$.
\end{abstract}

Key words: Harmony algorithm, bat algorithm, authentication system, biometric security, retina authentication, consuming

\section{INTRODUCTION}

Information security is worried about the affirmation of privacy, integrity and availability of information in all forms. There are many tools and techniques that can support the management of information security (Bhattacharyya et al., 2009).

An authentication process sets up the identity of some component under security. For example, a traveler authenticates herself/himself to a border guard by presenting a passport. Possession of the passport and resemblance to the attached photograph is considered sufficient proof that the traveler is the identified person. The act of validating the passport by checking a database of known passport serial numbers for the traveler is a form of authentication. Therefore, authentication is the process by which the identity of an entity is validated. Authenticating entities present credentials such as passwords or certificates as evidence of their identity. The entity is considered authentic if presented credentials are valid and sufficient. Note that authentication does not determine which entities should be given access but only verifies that an entity is who they claim to be. However, it is only after an entity is authenticated that their rights to resources can be assessed. Hence, failure to correctly authenticate users on the internet can leave online resources vulnerable to misuse.

A biometric system can provide two functions. One of which is verification and the other one is authentication. So, the techniques used for biometric authentication has to be stringent enough that they can employ both these functionalities simultaneously. As of now, subjective biometrics frameworks are being produced to utilize cerebrum reaction, facial acknowledgment and mental execution for seek at ports and high security zones (Havighurst, 2007).

The growing possibilities of modern communications and the explosive growth of information technology imposed special means of security (Mohammad and Al-Ta'i, 2018).

\section{Background}

Retina: The retina can be described as a layer of complex blood vessels and nerve cells on the back of the eye. Figure 1 shows a front view of the blood vessel pattern within the retina.

Corresponding Author: Iraq Ali Hussein, Department of Computer Science, Collage of Science, University of Diyala, Diyala, Iraq 


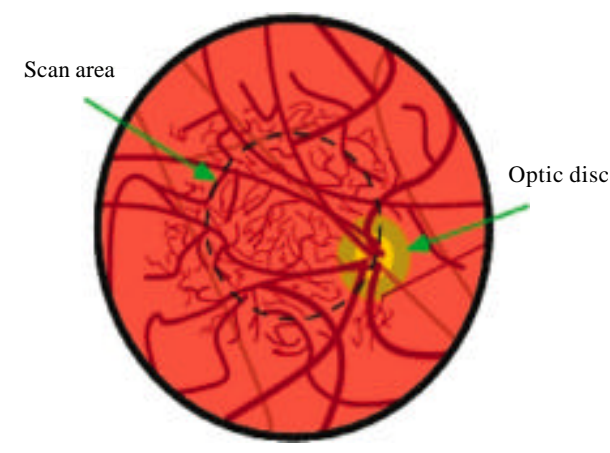

Fig. 1: Human retina

The retina is essentially sensory tissue consisting of multiple layers. The retina consists of millions of photoreceptors whose function is to gather the light rays that are sent to them and transform that light into electrical pulses that travel through the optic nerve into the brain which then converts these pulses into images. The two distinct types of photoreceptors that exist within the retina are called rods and cones. There are about 125 million rods in an eye which help tosee in low intensity lights and contribute in peripheral vision (Zibran, 2009).

Image preprocessing: Image pre-processing is the term for operations on images at the lowest level of abstraction. These operations do not increase image information content but they decrease it if entropy is an information measure (Bhattacharyya et al., 2009). The aim of pre-processing is an improvement of the image data that suppresses undesired distortions or enhances some image features relevant for further processing and analysis task (Miljkovic, 2009).

Canny processing: The purpose of edge detection in general is to significantly reduce the amount of data in an image while preserving the structural properties to be used for further image processing. Several algorithms exists and this worksheet focuses on a particular one developed by John F. Canny (JFC) in 1986 (Kaur and Ritika, 2013).

Detection: The probability of detecting real edge points should be maximized while the probability of falsely detecting non-edge points should be minimized. This corresponds to maximizing the signal-to-noise ratio.

Localization: The detected edges should be as close as possible to the real edges.

Number of responses: One real edge should not result in more than one detected edge (one can argue that this is implicitly included in the first requirement) (Kaur and Ritika, 2013).
Contrast processing: Image enhancement is a preprocessing step in many image processing applications. The aim of image enhancement is to improve the interpretability or perception of information in images for human viewers or to provide better input for other automated image processing techniques.

Contrast enhancement is one of the important research issues of image enhancement. A very popular technique for image enhancement is Histogram Equalization (HE). This system is generally utilized for picture improvement on account of its effortlessness and similarly better execution on a wide range of pictures (Kaur and Ritika, 2013).

The discrete Haar wavelet transform: An outstanding property of the Haar functions is that except function Haar $(0, t)$, the Haar function can be generated by the restriction of the ( $j-1$ ) the function to be half of the interval where it is different from zero, by multiplication with $\mathrm{p} 2$ and scaling over the interval $[0,1]$. These properties give considerable interest of the Haar function, since, they closely relate them to the wavelet theory. In this setting, the first two Haar functions are called the global functions while all the others are denoted as the local functions. Hence, the Haar function which is an odd rectangular pulse pair is the simplest and oldest wavelet (Porwik and Lisowska, 2004). A group of standard parameters has adopted. The results of parameters showed the proposed method has the highest security and integrity (Abed, 2017).

Literature review: During the last years, an increase is noticed in the research efforts toward developing systems dedicated to recognize humans through retinal images due to the increased need for a reliable system that is to identify the person stable with low error. Many researches appeared in the literature. These studies introduced different methods which vary in terms of processing time and efficiency. Some of the published works are listed in the following:

Morales et al. (2013) automatic detection of optic disc based on PCA and mathematical morphology, the goal is to facilitate the early detection of certain pathologies and to fully automate the process, so as to avoid specialist intervention. The method proposed for the extraction of the optic disc contour is mainly based on mathematical morphology along with Principal Component Analysis (PCA), it works properly on databases with a large degree of variability and improves the results of other state-ofthe-art methods, using Drions dataset, accuracy of their system is $94 \%$. 
Farzin et al. (2008) presented a novel retinal identification methods; it consists of three modules which are: vascular grids segmentation, feature extraction and matching stages. In the last stage, the modified correlation measureis used in order to obtain them similarity indexes between them feature vectors for each scale, then the totals valueof the likeness records is computed by summing scales weights closeness files. Their proposed method was evaluated on computerized, got recognizable identification accuracy rate was $99 \%$.

Barkhoda et al. (2009) proposed a new retina identification system using angular partitioning. The firsts stages of the proposed system is the preprocessing steps in which the extra margins of the retinal images are cropped and these boundary box of retina is extracted from the input retina to achieved translation invariants features. Then the cropped image is normalized to achieves the scale invariant and the adopted a similarity approach applied to extracts the vascular pattern. Then, the extracted vascular pattern is passed through a morphological thinning process. Their proposed system was tested on 360 images belong to DRIVE dataset, the achieved accuracy rate $98 \%$.

By Qamber et al. (2012) proposed a system for personal identification based on retinal blood vessels. At first, the enhancement stage is done using wavelet transform, then the vascular network is extracted by applying multilayer thresholding on the enhanced pattern. Next, the vessels endings and bifurcation points are extracted and used as feature points to establish a feature vector that based on distance and angles. Finally, matching process is performed using Mahalanobis distances. The proposed method had achieved an overall recognition rate $98.87 \%$.

Monisha and Seldevchristopher (2015) presented a robust method of human's authentication based on them retinal vascular patterns. First, top halt and bottom hat filtering are applied. Then, median filtering is applied repeatedly for smoothing, then the thresholding process is applied to segment the $\mathrm{m}$ vascular networks from the retinal image. After that thinning is performed to extract the skeleton of the blood vessels based on them morphological images analysis. A set of feature points (i.e., vascular bifurcation and ridge ending) is extracted using crossing number method. Finally, the Euclidean distance measure is used for matching between all the feature vectors. The proposed method was tested on DRIVE and STARE datasets. The attained recognition rate was $96 \%$ for STARE dataset, $97.5 \%$ for DRIVE dataset.

\section{MATERIALS AND METHODS}

Inspired algorithms: Algorithms from the fields of computational intelligence, biologically inspired computing and metaheuristics are applied to difficult problems, to which more traditional approaches may not be suited. These algorithms are called inspired algorithms. Inspired algorithms include: stochastic algorithms, evolutionary algorithms, physical algorithms, probabilistic algorithm, swarm algorithms, immune algorithms and neural algorithms (Michalewicz and Fogel, 2004).

Tow inspired algorithms are adopted in this study: bat (met heuristic algorithm) and harmony search (physical algorithm). The following inspired algorithms shown below.

Bat algorithm: Bat Algorithm (BA) is an inspired algorithm developed by Yang and $\mathrm{He}$ (2013) and BA has been found to be very efficient. The standard bat algorithm was based on the echolocation or bio-sonar characteristics of micro bats. Therefore, echolocation must be explained.

Echolocation of micro bats: There are about 1000 different species of bats [230]. Their sizes can vary widely, ranging from the tiny bumblebee bat of about $1.5-2 \mathrm{~g}$ to the giant bats with wingspan of about $2 \mathrm{~m}$ and may weigh up to about $1 \mathrm{~kg}$. Most bats uses echolocation to a certain degree among all the species, micro bats use echolocation extensively while mega bats do not. Echolocation is used to detect prey, avoid obstacles and locate their roosting crevices in the dark. Information security is becoming highly important because the huge of the WWW and it is one of the most important aspects of information and communication technology (Albayaty et al., 2018). Micro bats can emit a very loud sound pulse and listen for the echo that bounces back from the surrounding objects (Richardson, 2008).

Basic bat algorithm: Based on the above description and characteristics of bat echolocation (Yang and Deb, 2010) developed the bat algorithm with the following three idealized rules:

All bats use echolocation to sense distance and they also 'know' the difference between food/prey and background barriers in some magical way.

Bats fly randomly with velocity $v_{i}$ at position $x_{i}$ with a frequency fmin, varying wavelength $\lambda$ and loudness $\mathrm{A}_{0}$ to search for prey. They can automatically adjust the wavelength (or frequency) of their emitted pulses and adjust the rate of pulse emission $\mathrm{r} \epsilon[0,1]$, depending on the proximity of their target. Although, the loudness can vary in many ways, we assume that the loudness varies from a large (positive) $\mathrm{A} 0$ to a minimum constant value Amin Algorithm 1. 


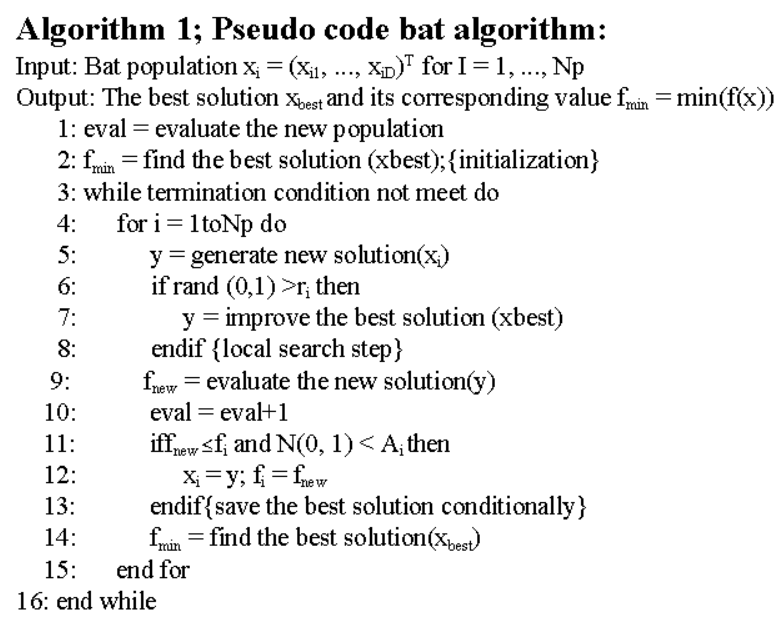

The Harmony Search (HS) algorithm: The music improvisation is a process of searching for the better harmony by trying various combinations of pitches that should follow any of the following three rules:

- $\quad$ Playing any one pitch from the memory

- Playing an adjacent pitch of one pitch from the memory

- $\quad$ Playing a random pitch from the possible range

The three rules in the HS algorithm are effectively directed using two essential parameters: Harmony Memory Considering Rate (HMCR) and Pitch Adjusting Rate (PAR). The steps of harmony search algorithm are (Wang et al., 2015):

Step 1; Parameters initialization: The optimization problem which is specified as in Eq. 1:

$$
\text { Find objective function } \mathrm{f}(\mathrm{x})
$$

where, $X_{j}=1,2, \ldots, N$. $f(x)$ is an objective function; $x$ the set of each decision variable $x_{j}$; Nthe Number of decision variables, $\mathrm{X}_{\mathrm{j}}$ the set of the possible range of values for each decision variable, that is $x_{j}$ min and $x_{j}{ }^{\max }$ are the lower and upper boundaries of the $\mathrm{jth}$ decision parameter, respectively.

Step 2; Harmony memory initialization and evaluation: A random initial population is generated such as in Eq. 2:

$$
x_{i, j}^{0}=x_{i}^{\min }+r_{i}\left(x_{j}^{\max }-x_{j}^{\min }\right)
$$

Where:

$$
\begin{aligned}
\mathrm{I} & =1,2,3, \ldots, \mathrm{HMS} \\
\mathrm{j} & =1,2, \ldots, \mathrm{N}
\end{aligned}
$$

$\mathrm{r}_{\mathrm{i}} \in[0,1]$ is a uniformly distributed random number generated new for each value of $i$. Solution vectors in HM are analyzed and their objective function values are then calculated.

Step 3; Improvisation: In this step, a new harmony vector is generated based on three rules, namely, memory consideration, pitch adjustment and random selection. The value of a design variable can be selected from the values stored in HM with a probability HMCR. It can be further, adjusted by moving to a neighbor value of a selected value from the HM with a probability of Pitch Adjusting Rate (PAR) or it can be selected randomly from the set of all candidate values without considering the stored values in HM with the probability of (1-HMCR).

Step 4; Harmony memory update: If the new harmony vector is better than the worst vector, based on the objective value and constraint violation, the new vector will replace the worst one.

Step 5; termination criterion check: HS algorithm is terminated if the stopping criterion (e.g. maximum number of improvisations) has been met. Otherwise, steps 3 and 4 are repeated. Algorithm 2 shows the pseudo code of harmony search algorithm (Wang et al., 2015).

The proposed method: This chapter describes the design and implementation of the proposed system. The proposed system is retina authentication system using intelligent algorithms (Bat and Harmony).

\author{
Algorithm 2; Pseudo code harmony algorithm: \\ Define objective function $f(x), x=\left(x_{1}, x_{2}, \ldots, x_{d}\right)^{T}$ \\ Define harmony memory accepting rate (raccept) \\ Define pitch adjusting rate and other parameters generate harmony memory \\ with random harmonies \\ While ( $\mathrm{t}<\mathrm{max}$ number of iterations) \\ While (is number of variables) \\ if (rand $<$ raccept), Choose a value from HM for the variable $i$ \\ if (rand $<$ pitch adjusting rate), adjust the value by adding \\ certain amount \\ End if \\ Else Choose a random value \\ End if \\ End while \\ Accept the new harmony (solution) if better \\ end while \\ Find the current best solution \\ End
}

The layout of the proposed system: The proposed system consists of two phases (testing and training), each of which consists of three stages (preprocessing, feature extraction, matching). These two phases share the first two stages (preprocessing and feature extraction) but they differ in third stage (matching). 


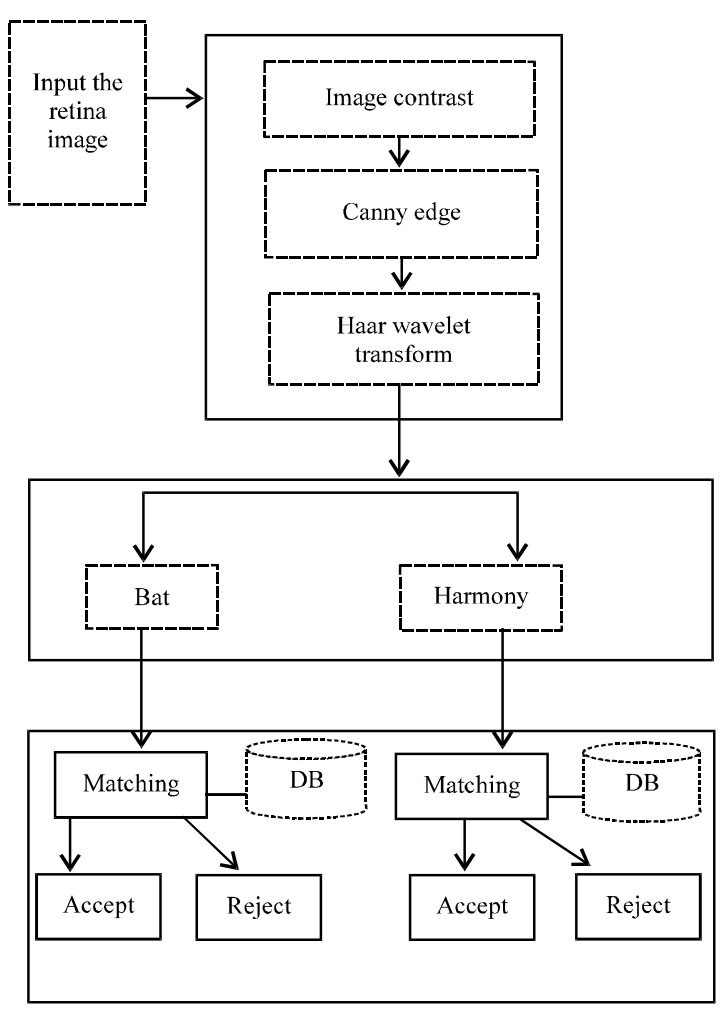

Fig. 2: Steps of testing

The proposed system begins with preprocessing stage that consists of the following steps:

- Enhancement of the image using the contrast filter

- Edge detection of the image using canny edge filter

- Transformation of the image using the Haar wavelet transformation

The second stage of the proposed system is the feature extraction in which three types of inspired algorithms are used:

- PSO algorithm

- Bat algorithm

- Harmony algorithm

- The general block diagram of the proposed system is shown in Fig. 2 and 3

Preprocessing stage: The preprocessing stage consists of three steps: contrast filter, canny edge filter and transformation using Haar wavelet transformation.

Contrast filtering: The first step of the preprocessing stage is contrasting the input retina image in order to enhance the image and to obtain more details of the

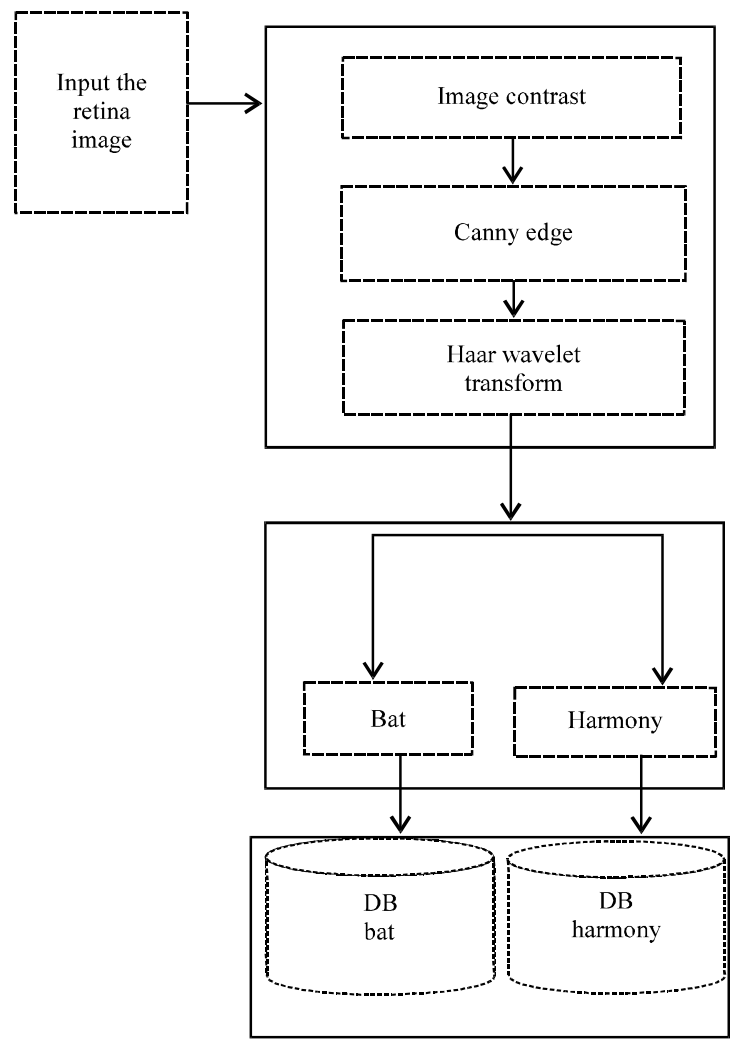

Fig. 3: Steps of training

vessel edge. Contrast enhancements improve the perceptibility of objects in the scene by enhancing the difference of the brightness between objects and their backgrounds as shown in Fig. 4.

Canny edge filtering: The second step of the preprocessing process is using canny filter in which it is used to detect the vessel edges and recognizes the edges in order to be ready for feature extraction. Canny filter takes the contrasted retina image and do the following processes on it:

- Gaussian filter

- Week edges

- Strong edge

- Final canny edges

Haar wavelet transformation: In this step, the resulted image from canny filter is transformed using Haar wavelet discrete transform. The output from this step is four sub images (LL, HL, LH, HH) as shown in Fig. 5.

Feature extraction: In this stage, a set of discriminating information is extracted from the final processed image. 
(a)

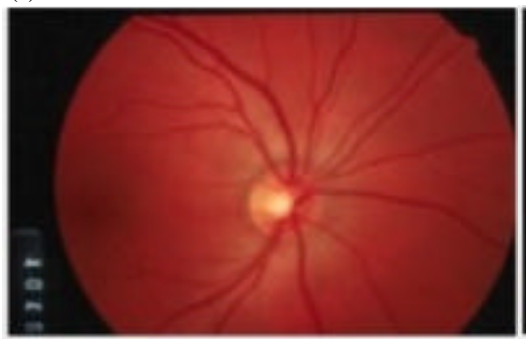

(b)

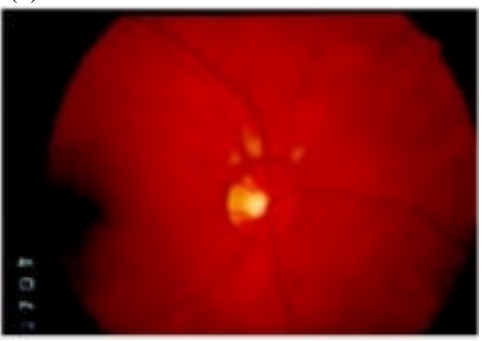

Fig. 4: Contrast filtering: a) Original image and b) After contrast
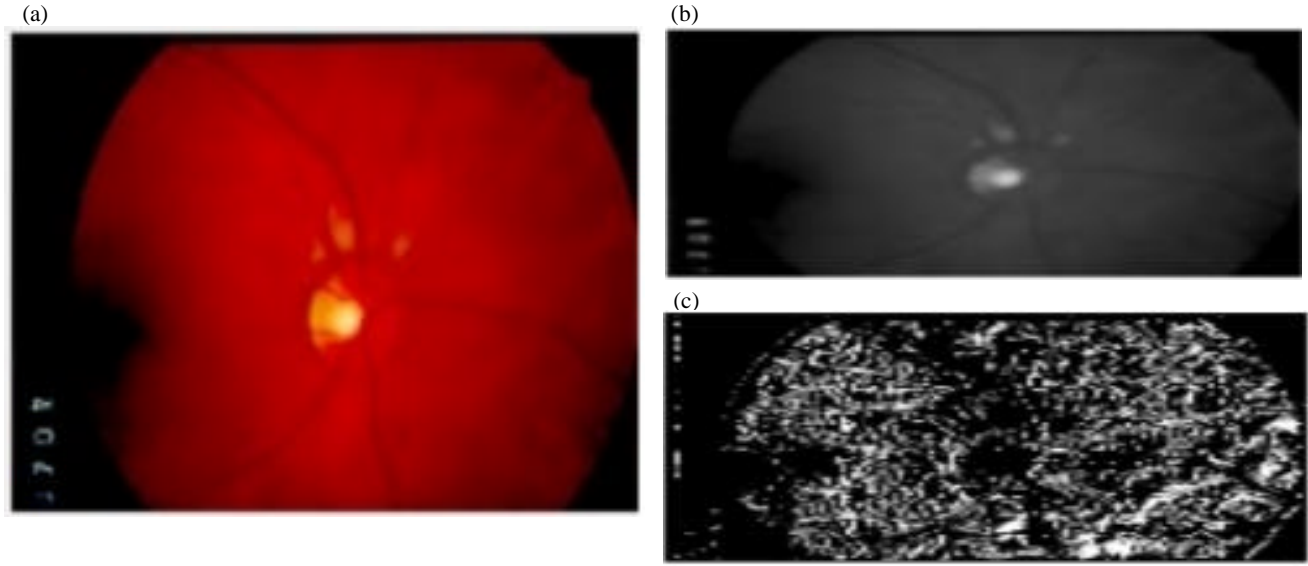

(d)

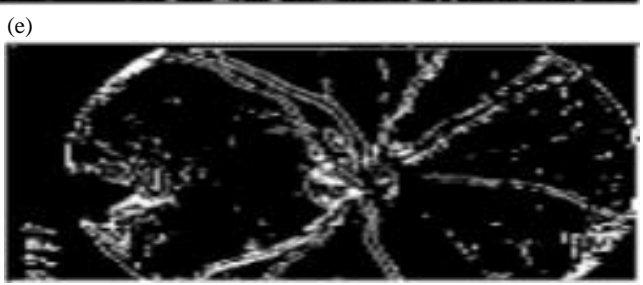

Fig. 5: Canny edge filter: a) Contrasted image; b) Canny edges (Gaussina filtered image); c) Weak edges; d) Strong edges and e) Final Canny edges

The extracted information represents the desired features vector in order to distinguish between persons. In the proposed system three different types of intelligent algorithms (Bat and Harmony) are used to extract the features from the retina image. In which each of these algorithms has its own characteristic.

Bat algorithm: Bat algorithm is an inspired algorithm which is used as an intelligent tool to extract features from the preprocessed retina image in the proposed system the implemented bat algorithm for features extraction in the proposed system is shown Algorithm 3.

\section{Algorithm 3; Bat algorithm for feature extraction:}

Input : Preprocessed retina image

Output: Features vector

$$
\{
$$

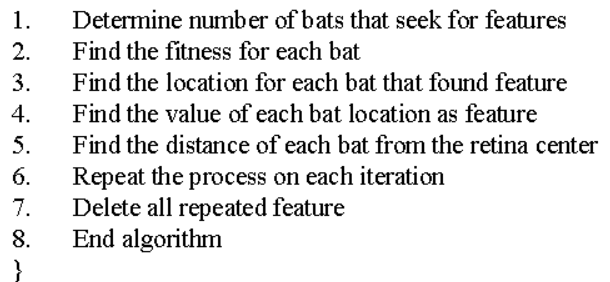

Harmony algorithm: Harmony algorithm is an inspired algorithm which is used as an intelligent tool to extract features from the preprocessed retina image in the proposed system. Depending on pseudo code of harmony search algorithm 2, the implemented harmony algorithm for features extraction in the proposed system is shown Algorithm 4. Note that, harmony algorithm works in four sub images (LL, HL, LH, HH) each with (100) iterations Table 1 and 2. 
Table 1: Initial parameters of bat

\begin{tabular}{lr}
\hline Parameters & Values \\
\hline Number of Variables (NVAR) & 128 \\
Number of inequality constraints (NG) & 6 \\
Number of equality constraints (NH) & 0 \\
Maximum number of iterations (MaxItr) & 100 \\
Harmony Memory Size (HMS) & 128 \\
Harmony Consideration Rate 0 HMCR $<1$ & 0.9 \\
Minimum Pitch Adjusting Rat (PARmin) & 0.1 \\
Maximum Pitch Adjusting Rate (PARmax) & 0.99 \\
Minimum bandwidth (bwmin) & 0.0001 \\
Maximum bandwidth (bwmax) & 1.0 \\
No. iteration & 100 \\
\hline
\end{tabular}

Table 2: Initial parameters of harmony

\begin{tabular}{lr}
\hline Parameters & Value \\
\hline Parameter denotes loudness (A) & 0.7 \\
Denotes Population size (NP) & 30 \\
Parameter denotes pulse rate & 0.3 \\
Parameter denotes frequency minimum (Qmin) & -15 \\
Parameter denotes frequency maximum (Qmax) & 15 \\
Denotes Lower bound (Lower) & 0 \\
Denotes Upper bound (Upper) & 2 \\
No. of iteration & 100 \\
\hline
\end{tabular}

Algorithm 4; Implemented harmony search algorithm: Input: Preprocessed retina image

Output: Features set \{

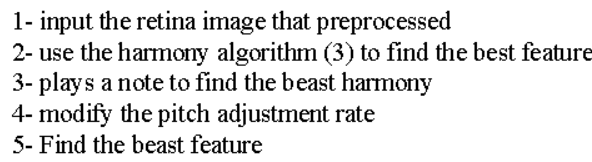

Matching: Feature matching is the most important part of any biometric system. It is used to find the degree of similarity between two retina patterns, the distance is computed between the features vector of the template stored in the DB and of the tested image.

In our proposed system, we use 3 inspired algorithm, so, each algorithm has its awn database, the set of feature

(b)
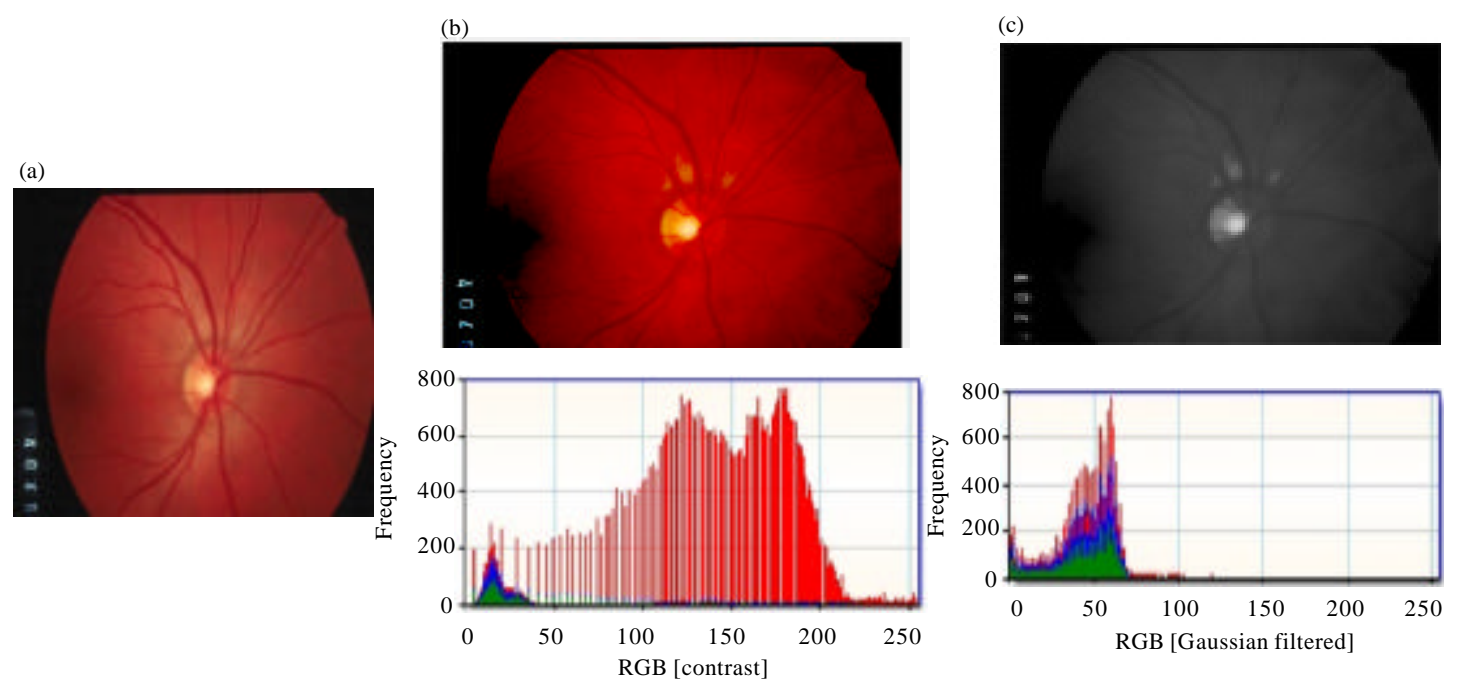

vector will save in the database in training section to be matching between set feature vector in test section. The measures used for the proposed system are "Euclidean distance (Sum of Squared Difference SSD) (Algorithm 5).

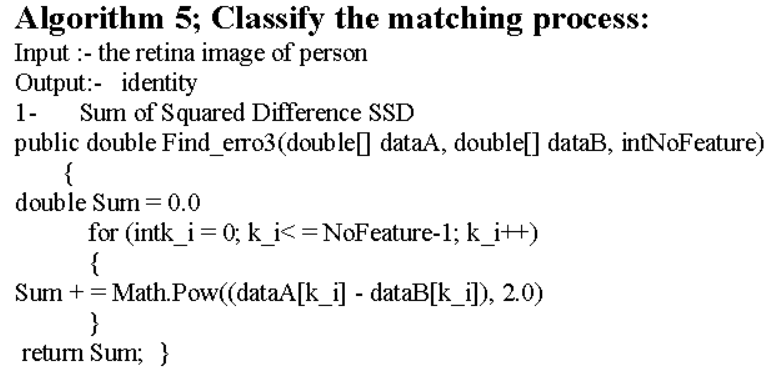

\section{RESULTS AND DISCUSSION}

This chapter presents the results of the proposed system with its evaluation. The proposed system was implemented under the environment: Windows (8.1) operating system, HP laptop computer (processor: Intel Core 5, CPU: $4 \mathrm{GHz}$ and RAM: $4 \mathrm{~GB}$ ) and programming language is (VB.NET) (Table 3-9).

The Dirons-DB data set has been used to test the performance of the developed system. The Dirons-DB dataset contains (110) images in which the size of the each image is $(565 \times 584)$ pixels. These images are divide as: (100) images for training and (10) images for testing. The 110 images have been used for testing the identification/verification performance. Each one of the 110 original images was subjected to two levels of brightness re-distributions. So, one retina image had produced as variants representing each retina sample belong to certain individual. Thus, a dataset consists of 110 simulated images Fig. 6 and 7.

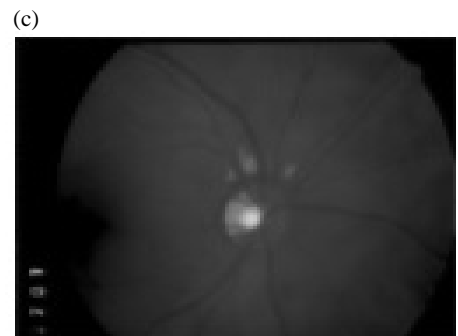

Fig. 6: The result of preprocessing: a) Image; b) Contrast and c) Gaussian 

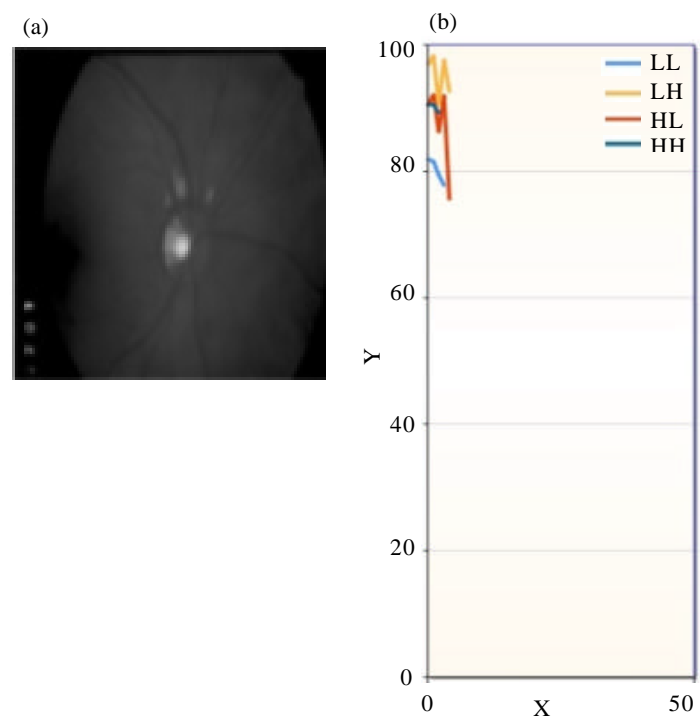

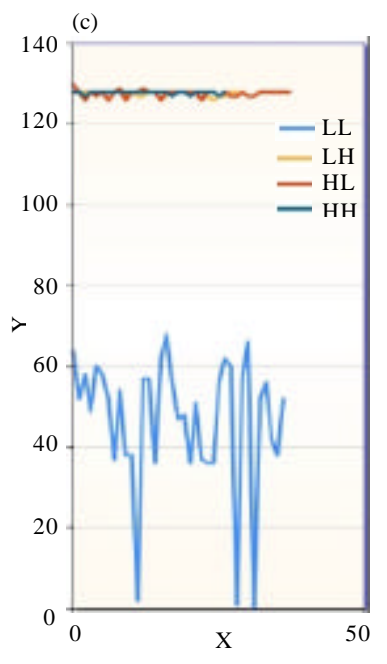

Fig. 7: The results of histograms for the extracted features with the tow inspired algorithms: a) Gaussian image; b) Contrast and c) Harmony

Table 3: Mean and standard deviation values for the tow inspired algorithms for three images

\begin{tabular}{|c|c|c|c|}
\hline \multicolumn{4}{|l|}{ Bat algorithm } \\
\hline Mean & \multicolumn{3}{|c|}{ SD } \\
\hline 15.9802289285714 & & \multicolumn{2}{|c|}{180182852034036} \\
\hline 17.6128916923077 & & \multicolumn{2}{|c|}{20.2815419610354} \\
\hline 27.5987845 & & \multicolumn{2}{|c|}{25.5670983247984} \\
\hline Algorithms/No. iteration & Time min & Time max & Time mean \\
\hline \multicolumn{4}{|l|}{ Bat } \\
\hline 100 & $00: 00: 6.6$ & $00: 00: 8.0$ & $00: 00: 7.3$ \\
\hline 200 & $00: 00: 8.7$ & $00: 00: 11.1$ & $00: 00: 9.9$ \\
\hline 300 & $00: 00: 10.4$ & $00: 00: 12.0$ & $00: 00: 11.3$ \\
\hline \multicolumn{4}{|l|}{ Harmony } \\
\hline 100 & $00: 00: 3.0$ & $00: 00: 3.0$ & $00: 00: 3.0$ \\
\hline 200 & $00: 00: 4.4$ & $00: 00: 5.4$ & $00: 00: 4.9$ \\
\hline 300 & $00: 00: 6.0$ & $00: 00: 6.8$ & $00: 00: 6.4$ \\
\hline
\end{tabular}

Table 5: FAR, FRR and the accuracy versus different sigma values for the bat method

\begin{tabular}{lclc}
\hline Parameters & FAR & FRR & Accuracy \\
\hline 0.5 & 0.013 & 0.0133 & 98.66 \\
1 & 0.014 & 0.0139 & 98.59 \\
1.5 & 0.015 & 0.027 & 97.96 \\
2 & 0.028 & 0.041 & 96.53 \\
\hline
\end{tabular}

Table 6: FAR, FRR and the accuracy versus different threshold values for the harmony method

\begin{tabular}{lllc}
\hline Parameters & FAR & FRR & Accuracy \\
\hline 0.5 & 0.027 & 0.013 & 99.98 \\
1 & 0.080 & 0.067 & 99.93 \\
1.5 & 0.200 & 0.173 & 99.81 \\
2 & 0.183 & 0.200 & 99.80 \\
\hline
\end{tabular}

Table 7: The FAR and FRR and accuracy for the three inspired algorithm

\begin{tabular}{llcc}
\hline Algorithm & FAR $(\%)$ & FRR $(\%)$ & Accuracy \\
\hline Harmony & 0.027 & 0.013 & 99.98 \\
Bat & 0.133 & 0.200 & 98.65 \\
\hline
\end{tabular}

0.133

0.200
Harmony algorithm

\begin{tabular}{ll}
\hline Mean & SD \\
41.1914418583334 & 13.5909131618093 \\
41.6883777333333 & 13.6108904842375 \\
38.240457203252 & 14.7362790510825 \\
\hline
\end{tabular}

Table 8: The TPR and FPR and accuracy of tow proposed algorithm

\begin{tabular}{llll}
\hline Algorithm & TPR $(\%)$ & FPR $(\%)$ & Accuracy (\%) \\
\hline Bat & 0.986 & 0.014 & 98.65 \\
Harmony & 1.000 & 0.013 & 99.98 \\
\hline \multicolumn{3}{l}{ Table 9: Comparison proposed sy stem with previous studies } \\
\hline \multicolumn{3}{l}{ Algorithm } & Accuracy (\%) \\
\hline Morales S. (Barkhoda et al., 2009) & 94 \\
Frazin et al. (Qamber et al., 2012) & 99 \\
Barkhoda et al. (Monisha Seldevchristopher, 2015) & 98 \\
Qamber et al. (Michalewicz and Fogel, 2004) & 98.87 \\
Monisha et al. (Richardson, 2008) & 96 \\
Manjiri. B & & 98 \\
M Islamuddin Ahmed & 84.2 \\
Harmony & & 99.98 \\
Bat & & 98.65 \\
\hline
\end{tabular}

\section{CONCLUSION}

Among several available biometric system, retina recognition are said to be the most secure system and stable because the vascular patterns are unique and it is hard to be lost or making a copy of it. In this study, an efficient and simple method is proposed for individual recognition system based on retinal blood vessels. In comparison with other retina recognition methods appeared in the literature. The introduced system proposed a simple module for rotation compensation. Also, the concept of harmony and bats algorithms as 
discrimination feature. The results of the tests conducted on the public dataset (i.e., Drions) indicate that the performance of the proposed method is too high (i.e., $100 \%$ recognition rate) for both dataset.

\section{REFERENCES}

Abed, H.N., 2017. Robust and secured image steganography using LSB and encryption with QR code. J. AL. Qadisiyah Comput. Sci. Math., 9: 1-9.

Albayaty, I.S., A.L. Ahmed, N.H. Hassoon and H.N. Abed, 2018. Hiding information in an image based on bats algorithm. Iraqi J. Inf. Technol., 8: 128-141.

Barkhoda, W., F.A. Tab and M.D. Amiri, 2009. Rotation invariant retina identification based on the sketch of vessels using angular partitioning. Proceedings of the 2009 International Multi Conference on Computer Science and Information Technology, October 12-14, 2009, IEEE, Mragowo, Poland, ISBN: 978-1-4244-5314-6, pp: 3-6.

Bhattacharyya, D., R. Ranjan, F. Alisherov and M. Choi, 2009. Biometric authentication: A review. Int. J. UE Serv. Sci. Technol., 2: 13-28.

Farzin, H., H. Abrishami-Moghaddam and M.S. Moin, 2008. A novel retinal identification system. EURASIP. J. Adv. Signal Process., 2008: 1-10.

Havighurst, R., 2007. User Identification and Authentication Concepts. In: Mechanics of user Identification and Authentication: Fundamentals of Identity Management, Todorov, D. (Ed.). Auerbach Publications, Boca Raton, Florida, USA., ISBN:978-1-4200-5219-0, pp: 1-64.

Kaur, S. and Ritika, 2013. Contrast enhancement techniques for images-a visual analysis. Intl. J. Comput. Appl., 64: 20-25.

Michalewicz, Z. and D.B. Fogel, 2004. How to Solve it: Modern Heuristics. 2nd Edn., Springer, Berlin, Germany, ISBN:978-3-540-22494-5, Pages: 554.

Miljkovic, O., 2009. Image pre-processing tool. Kragujevac J. Math., 32: 97-107.
Mohammad, I.R. and Z.T.M. Al-Ta'i, 2018. Image steganography based the behavior of particle swarm optimization. J. Theor. Appl. Inf. Technol., 96: 3696-3706.

Monisha, L.S.L. and C. Seldevchristopher, 2015. Biometric identification using retina scan. Intl. J. Adv. Res. Trends Eng. Technol., 2: 145-151.

Morales, S., V. Naranjo, J. Angulo and M. Alcaniz, 2013. Automatic detection of optic disc based on PCA and mathematical morphology. Med. Imaging IEEE. Trans., 32: 786-796.

Porwik, P. and A. Lisowska, 2004. The haar-wavelet transform in digital image processing: Its status and achievements. Mach. Graphics Vision, 13: 79-98

Qamber, S., Z. Waheed and M.U. Akram, 2012. Personal identification system based on vascular pattern of human retina. Proceedings of the 2012 Cairo International Conference on Biomedical Engineering (CIBEC), December 20-22, 2012, IEEE, Giza, Egypt, ISBN:978-1-4673-2800-5, pp: 64-67.

Richardson, P., 2008. Bats. Natural History Museum, London, England, UK.,

Wang, X., X.Z. Gao and K. Zenger, 2015. An Introduction to Harmony Search Optimization Method. Springer, Berlin, Germany, Pages: 88.

Yang, X.S. and S. Deb, 2010. Eagle Strategy Using Levy Walk and Firefly Algorithms for Stochastic Optimization. In: Nature Inspired Cooperative Strategies for Optimization (NICSO 2010), Gonzalez, J.R., D.A. Pelta, C. Cruz, G. Terrazas and N. Krasnogor (Eds.). Springer, Berlin, Germany, ISBN: 978-3-642-12537-9, pp: 101-111.

Yang, X.S. and X.S. He, 2013. Bat algorithm: Literature review and applications. Int. J. Bio-Inspired Comput., 5: 141-149.

Zibran, M.F., 2009. Eye based authentication: Iris and retina recognition. Master Thesis, Department of Computer Science, University of Saskatchewan, Saskatoon, Canada. 